\title{
TANGENT SPACES AND GROMOV-HAUSDORFF LIMITS OF SUBANALYTIC SPACES
}

\author{
ANDREAS BERNIG AND ALEXANDER LYTCHAK
}

\begin{abstract}
It is shown that the Gromov-Hausdorff limit of a subanalytic 1-parameter family of compact connected sets (endowed with the inner metric) exists. If the family is semialgebraic, then the limit space can be identified with a semialgebraic set over some real closed field. Different notions of tangent cones (pointed Gromov-Hausdorff limits, blowups and Alexandrov cones) for a closed connected subanalytic set are studied and shown to be naturally isometric. It is shown that geodesics have well-defined Euclidean directions at each point.
\end{abstract}

\section{INTRODUCTION}

The length metric on subanalytic spaces is far from being understood. For instance, Hardt's conjecture from 1983 ([11]), claiming that the metric is a subanalytic function, remains open. Only after the introduction of Lregular decompositions by Kurdyka ([12]) and Parusiński ([17]), there has been some progress in understanding the metric structure of subanalytic sets (e.g. [10], [4], [13], [16], [8], [18], [1], [2]). However, it seems that many natural questions are still out of reach.

In this note, we provide results concerning the local structure of subanalytic spaces. Our local results rely on the following global theorem.

THEOREM 1.1. Let $X \subset \mathbb{R} \times \mathbb{R}^{n}$ be a compact subanalytic 1-parameter family of subsets of $\mathbb{R}^{n}$. Suppose that each fiber $X_{t}:=X \cap\left(\{t\} \times \mathbb{R}^{n}\right)$ is connected. Then the Gromov-Hausdorff limit $\lim _{t \rightarrow 0^{+}}\left(X_{t}, d_{X_{t}}\right)$ exists.

Considering for a single subanalytic space $X$ the family $\frac{1}{t} X$ we obtain that the tangent cone $\lim _{t \rightarrow 0}\left(X, \frac{1}{t} d_{X}, x\right)$ of $X$ with respect to the inner metric $d_{X}$ exists at each point $x \in X$. However, the proof of Theorem 1.1 provides not only an abstract convergence of isometry classes of spaces but gives us an explicit metric space in the limit. This allows us to speak about the tangent space and about differentiability of maps. The next theorem is our main result concerning the infinitesimal geometry of subanalytic sets.

1991 Mathematics Subject Classification. 53C22, 32B20.

Key words and phrases. Tangent spaces, Gromov-Hausdorff convergence, subanalytic sets, geodesics.

The first author was supported by Deutsche Forschungsgemeinschaft grant BE 2484/2- 
THEOREM 1.2. Let $X \subset \mathbb{R}^{n}$ be a closed, connected subanalytic set, endowed with the inner metric $d_{X}$. Then the Gromov tangent space $T_{x} X$ is naturally isometric to the Alexandrov cone $C_{x} X$, i.e. each blow up $X_{x}^{\left(t_{i}\right)}$ is naturally isometric to $C_{x} X$. Moreover, these tangent spaces are Euclidean cones. In particular, the angle between two geodesics starting at $x$ is welldefined. Each subanalytic Lipschitz map $f:(X, x) \rightarrow(Y, y)$ is differentiable at $x$ in the metric sense.

In general, Gromov-Hausdorff convergence is too weak and it does not allow to compare the topology of the limit space with the topology of the elements of the convergent sequence. For example collapsing can occur, i.e. the dimension of the tangent space $T_{x} X$ may be smaller than the dimension of each neighborhood of $x$ in $X$. However some structure survives in $T_{x} X$. Recall that the density of a $k$-dimensional subanalytic set $X$ at a point $x \in X$ is given by $\theta(X, x):=\lim _{r \rightarrow 0} \frac{\mathcal{H}^{k}\left(B_{r}(x)\right)}{b_{k} r^{k}}$ where $b_{k}$ is the volume of the $k$-dimensional unit ball. The existence of the limit is proven in [14]. We prove

PROPOSITION 1.3. $\quad$ a) The unit sphere in $T_{x} X$ is connected if and only if this is the case for small spheres $S_{r}(x)$ in $X$.

b) The density of $X$ at $x$ equals the ratio of the Hausdorff measure of the unit ball in $T_{x} X$ and the volume $b_{k}$ of the $k$-dimensional Euclidean ball.

Each subanalytic subset $X \subset \mathbb{R}^{n}$ defines at each point $x \in X$ the so called subanalytic tangent cone $T_{x}^{s u b} X \subset \mathbb{R}^{n}$ (which is the same as the tangent cone of the metric space $\left(X, d_{e}\right)$, where $d_{e}$ is the Euclidean metric on $X$ ). In general, $T_{x}^{s u b}$ contains less information than the metric tangent cone $T_{x} X$, but they are closely related:

THEOREM 1.4. Let $X$ be as above. Then the identity $\iota:\left(X, d_{X}\right) \rightarrow$ $\left(X, d_{e}\right)$ is differentiable at $x$. The differential $D_{x} \iota: T_{x} X \rightarrow T_{x}^{s u b} X$ is a 1-Lipschitz homogeneous map that preserves the distances to the origin and lengths of arbitrary curves. Moreover each point $v \in T_{x}^{s u b} X$ has at most $m=m(X)$ preimages.

The known results about the inner metric structure of subanalytic sets are too weak to exclude strange behavior (like oscillations) of geodesics. One of the keys for the proof of the above results is a regularity result for geodesics. We show that each geodesic in $\left(X, d_{X}\right)$ has a well defined Euclidean direction at each point.

Remark 1.1. Theorem 1.1 and Theorem 1.4 are valid for every o-minimal structure (see [19] for o-minimal structures). . Theorem 1.2 and Proposition 1.3 are valid for every polynomially bounded o-minimal structure.

In the last section, we will study the semialgebraic case. It turns out that every closed, rationally bounded semialgebraic set $S$ in $R^{n}$, where $R$ 
is any real closed field, defines a compact inner metric space $\left(\bar{S}, \bar{d}_{S}\right)$. The Gromov-Hausdorff limit of a semialgebraic 1-parameter family of such spaces is again semialgebraic, the limit space is the fiber over the point $0^{+}$in the real spectrum of $R[t]$.

THEOREM 1.5. Let $R$ be a real closed field. Let $X \subset R \times R^{n}$ be a semialgebraic 1-parameter family of subsets of $R^{n}$. Suppose that $X \subset B_{a}(0)$ for some natural number $a$ and that each fiber $X_{t}:=X \cap\left(\{t\} \times R^{n}\right)$ is semialgebraically connected. Let $S:=X_{0^{+}}$be the fiber over $0^{+} \in S p e c_{r} R[t]$. Then $\lim _{t \rightarrow 0^{+}}\left(\bar{X}_{t}, \bar{d}_{X_{t}}\right)=\left(\bar{S}, \bar{d}_{S}\right)$.

Remark that $S$ is again semialgebraic, but over the real closed field $k\left(0^{+}\right)$, which is the field of algebraic Puiseux series over $R$. The analogous convergence result for the induced metrics on the fibers $X_{t}$ instead of the inner metrics was known to be true for some while, see [7].

The main technical ingredient in our proofs is a decomposition, due to Kurdyka and Orro ([13]), of a given subanalytic set into subanalytic pieces such that inner and Euclidean metric differ only by a factor near 1. For further information concerning the inner metric on subanalytic or more general stratified spaces, we refer the reader to [18] and [16]. A detailed study of geodesic metric spaces can be found in [6] and [9].

\section{TANGent CONES OF MEtRiC AND SUbanalytic SPACES}

2.1. Notations. For a metric space $(X, d)$ we will denote by $d_{X}$ the inner metric on $X$ (which can be infinite if there is no rectifiable path between two points). The identity $i d:\left(X, d_{X}\right) \rightarrow(X, d)$ is 1-Lipschitz and preserves lengths of curves. A geodesic in a metric space $(X, d)$ is an isometric embedding $\gamma:[a, b] \rightarrow X$ of an interval. Subsets of metric spaces will always be considered with the induced metric, if not otherwise stated.

By $B_{r}(x)$ we will denote the closed metric ball of radius $r$ around $x$ and by $r X$ the metric space $(X, r d)$.

2.2. Metric cones. Compare [15] for more on metric cones. A metric cone is a pointed metric space $(X, d, x)$ together with a (pointwise) continuous family $\delta_{t}, t \in \mathbb{R}^{+}$of maps (dilatations) $\delta_{t}:(X, x) \rightarrow(X, x)$, such that $d\left(\delta_{t}(y), \delta_{t}(z)\right)=t d(y, z)$ and $\delta_{t} \circ \delta_{s}=\delta_{s t}$. A map between metric cones is homogeneous if it commutes with the dilatations. A metric cone $(T, 0)$ is called radial if for each $x \in T$ with $d(x, 0)=1$ the map $t \rightarrow \delta_{t}(x)$ is a ray, i.e. if $d\left(\delta_{t}(x), \delta_{s}(x)\right)=|t-s|$. If $(T, 0)$ is a radial cone we can consider $S=\{x \in T \mid d(x, 0)=1\}$ the unit sphere in $T$ and the Euclidean cone $C S$ (compare [9]) over $S$. Then the natural map $F: C S \rightarrow T$ that sends $t x$ to $\delta_{t}(x)$ is homogeneous and bilipschitz.

2.3. Ultralimits and blow-ups. See [6] and [15] for more details. We will use a fixed non-principal ultrafilter $\omega$ on the set of natural numbers. For pointed metric spaces $\left(X_{i}, x_{i}\right)$ we will denote their ultralimit by $\lim _{\omega}\left(X_{i}, x_{i}\right)$. 
Remark that if the isometry classes of proper spaces $\left(X_{i}, x_{i}\right)$ build a relatively compact set with respect to the pointed Gromov-Hausdorff topology, then the $\operatorname{limit}_{\omega} \lim _{\omega}\left(X_{i}, x_{i}\right)$ is a proper space and its isometry class is a pointed Gromov-Hausdorff limit of a subsequence of $\left(X_{i}, x_{i}\right)$.

For a fixed metric space $(X, x)$ and a zero sequence $\left(t_{i}\right) \rightarrow 0$ we consider the ultralimit $\lim _{\omega}\left(\frac{1}{t_{i}} X, x\right)$, denote it by $X_{x}^{\left(t_{i}\right)}$ and call it the blow-up of $X$ at $x$ at the scale $\left(t_{i}\right)$. The base point of the blow-ups will be denoted by 0 .

If $X$ is proper, and if the pointed Gromov-Hausdorff $\operatorname{limit}_{t \rightarrow 0}\left(\frac{1}{t} X, x\right)$ exists, then all the blow-ups $X_{x}^{\left(t_{i}\right)}$ are in the isometry class of $\lim _{t \rightarrow 0}\left(\frac{1}{t} X, x\right)$.

If $f:(X, x) \rightarrow(Y, y)$ is an $L$-Lipschitz map, then for each sequence $\left(t_{i}\right) \rightarrow$ 0 there is an induced $L$-Lipschitz blow-up $f_{x}^{\left(t_{i}\right)}:\left(X_{x}^{\left(t_{i}\right)}, 0\right) \rightarrow\left(Y_{y}^{\left(t_{i}\right)}, 0\right)$.

2.4. Tangent cones and differentials. We refer the reader to [15] for a detailed study of differential properties of general metric spaces.

Let $X$ be a metric space, $x \in X$. We say that a metric cone $(T, 0)$ is the tangent cone $T_{x} X$ at $x$, if for each zero sequence $\left(t_{i}\right)$ an isometry $\tau^{\left(t_{i}\right)}:(T, 0) \rightarrow\left(X_{x}^{\left(t_{i}\right)}, 0\right)$ is fixed, such that for each $s>0$ and each point $p \in T$ the point $\tau^{\left(s t_{i}\right)}\left(\delta_{s}(p)\right) \in X_{x}^{\left(s t_{i}\right)}$ coincides with $\tau^{\left(t_{i}\right)}(p) \in X_{x}^{\left(t_{i}\right)}$ if the sets $X_{x}^{\left(t_{i}\right)}$ and $X_{x}^{\left(s t_{i}\right)}$ are identified in the natural way.

Remark 2.1. The definition implies that all the blow-ups of $X$ at $x$ are isometric and a fixed metric space $(T, 0)$ in the isometry class of the blowups is fixed. The commutation relations required in the definition are always satisfied, if the isometries $\tau^{\left(t_{i}\right)}$ are given in some "natural" way.

If for metric spaces $(X, x)$ and $(Y, y)$ the tangent cones $T_{x} X$ and $T_{y} Y$ exist, we say that a Lipschitz map $f:(X, x) \rightarrow(Y, y)$ is differentiable at $x$ if for each zero sequence $\left(t_{i}\right)$ the blow-up $f_{x}^{\left(t_{i}\right)}$ considered as a map from $T_{x} X$ to $T_{y} Y$ does not depend on the sequence $\left(t_{i}\right)$. This unique blow-up is in this case a homogeneous Lipschitz map. It will be denoted by $D_{x} f$ and called the differential of $f$ at $x$. In particular a Lipschitz curve $\gamma:[0, a) \rightarrow X$ starting at $x$ is differentiable at 0 iff the point $v=\left(\gamma\left(t_{i}\right)\right) \in X_{x}^{\left(t_{i}\right)}=T_{x} X$ is independent of the zero sequence $t_{i}$. In this case the differential $D_{0} \gamma$ : $T_{0}([0, a))=[0, \infty) \rightarrow T_{x} X$ is given by $D_{0} \gamma(t)=\delta_{t}(v)$. We will identify $v$ with $D_{0} \gamma$.

2.5. Alexandrov cone. By $\Gamma_{x}$ we denote the set of all geodesics starting at $x$. On $\Lambda_{x}:=\Gamma_{x} \times[0, \infty)$ we consider the pseudo metric given by $d\left(\left(\gamma_{1}, s_{1}\right),\left(\gamma_{2}, s_{2}\right)\right):=\lim \sup _{t \rightarrow 0} \frac{d\left(\gamma_{1}\left(s_{1} t\right), \gamma_{2}\left(s_{2} t\right)\right)}{t}$ and call its completion the Alexandrov cone $C_{x}\left(=C_{x} X\right)$. The points $(\gamma, 0) \in \Gamma_{x} \times[0, \infty)$ are identified to the origin 0 in $C_{x}$ and for $t \in \mathbb{R}^{+}$the maps $\delta_{t}: C_{x} \rightarrow C_{x}$ are $t$-dilatations, that define the structure of a radial metric cone on $C_{x}$.

For each zero sequence $\left(t_{i}\right) \rightarrow 0$ the natural 1-Lipschitz map $\exp _{x}^{\left(t_{i}\right)}$ : $\Gamma_{x} \times[0, \infty) \rightarrow X_{x}^{\left(t_{i}\right)}$ defined by $\exp _{x}^{\left(t_{i}\right)}((\gamma, s)):=\left(\gamma\left(s t_{i}\right)\right)$ uniquely extends to a 1-Lipschitz map $\exp _{x}^{\left(t_{i}\right)}: C_{x} \rightarrow X_{x}^{\left(t_{i}\right)}$. 
Let $x$ be a point in $X$. Then all the exponential maps $\exp _{x}^{\left(t_{i}\right)}$ are isometric embeddings iff the limes superior in the definition of the metric on $C_{x}$ is a limes. The upper angle and the lower angle between each pair of geodesics starting at $x$ coincide iff in addition $C_{x}$ is a Euclidean cone.

2.6. Subanalytic tangent cone. Let $T_{x}^{s u b} X$ denote the subanalytic tangent cone of $X$ at $x$, i.e. the set

$T_{x}^{s u b} X:=\left\{v \in \mathbb{R}^{n}: \forall \epsilon>0 \exists y \in X \exists \lambda \in[0, \infty):\|y-x\|<\epsilon,\|\lambda(y-x)-v\|<\epsilon\right\}$

By the curve selection lemma, this is the same as the set of initial vectors of continuous subanalytic curves starting at $x$ and contained in $X$. Note that $T_{x}^{s u b} X$ is a subanalytic cone ([14]).

Remark 2.2. The cone $T_{x}^{s u b} \subset \mathbb{R}^{n}$ equals the (metric) tangent cone to the metric space $\left(X, d_{e}\right) \subset \mathbb{R}^{n}$ at $x$. A Lipschitz curve $\gamma:[0, \epsilon) \rightarrow X$ starting at $x$ is differentiable as a map to $\left(X, d_{e}\right)$, iff $\gamma$ is differentiable at 0 as a curve in $\mathbb{R}^{n}$. A subanalytic map $f:(X, x) \rightarrow(Y, y)$ that is Lipschitz with respect to the induced metric defines a homogeneous Lipschitz differential $D_{x}^{s u b} f: T_{x}^{s u b} X \rightarrow T_{y}^{s u b} Y$.

\section{Gromov-Hausdorff limit in a subanalytic family}

This section is devoted to the proof of Theorem 1.1.

Proof. Let $X \subset \mathbb{R} \times \mathbb{R}^{n}$ be a compact subanalytic set such that $X_{t}:=$ $X \cap(\{t\} \times \mathbb{R})$ is connected.

We use the known fact ([13]) that for each $C>1$ the set $X$ can be decomposed as finite union $X=\cup_{i=1}^{m} X^{i}$ such that each fiber $X_{t}^{i}$ is subanalytic, compact, connected with the property that length and induced metric on $X_{t}^{i}$ differ by at most a factor $C$.

It follows immediately that the diameters of the metric spaces $\left(X_{t}, d_{X_{t}}\right), t \in$ $\mathbb{R}$ are uniformly bounded from above by some $0<D<\infty$.

It also follows that the family is equicompact, i.e. for each $\epsilon>0$, there exists $N_{1}(\epsilon)$, independent of $t$, such that each $X_{t}$ can be covered by at most $N_{1}(\epsilon)$ balls of radius $\epsilon$. Equivalently, there exists $N_{2}(\epsilon)$ such that each $\epsilon$ separated net in $X_{t}$ contains at most $N_{2}(\epsilon)$ points.

Consider two subanalytic curves $\gamma_{1}, \gamma_{2}:(0, \epsilon) \rightarrow X$ with $\gamma_{i}(t) \in X_{t}$. We claim that the limit $\lim _{t \rightarrow 0^{+}} d_{X_{t}}\left(\gamma_{1}(t), \gamma_{2}(t)\right) \in[0, \infty)$ exists. This follows from the theorem of Kurdyka-Orro ([13]) stating that for each $\eta>0$ there exists a subanalytic distance $\tilde{d}: X \times X \rightarrow \mathbb{R}$ such that $\tilde{d}(x, y) \leq d_{X_{t}}(x, y) \leq$ $(1+\eta) \tilde{d}(x, y)$ for all $x, y \in X_{t}$. Since the $\operatorname{limit}_{\lim } \lim _{t \rightarrow 0^{+}} \overline{\tilde{d}}\left(\gamma_{1}(t), \gamma_{2}(t)\right)$ exists in $[0,2 D]$ (by properties of subanalytic functions), we obtain that $\lim _{t \rightarrow 0^{+}} d_{X_{t}}\left(\gamma_{1}(t), \gamma_{2}(t)\right) \in[0,2 D]$ exists.

On the space $\Lambda^{\text {sub }}$ of subanalytic curve germs $\gamma:(0, \epsilon) \rightarrow X$ with $\gamma(t) \in$ $X_{t}$ this limit defines a pseudo-metric $d_{\text {lim }}$.

Let $\gamma_{1}, \ldots, \gamma_{k}$ be an $\epsilon$-separated net in $\Lambda^{s u b}$. Then, for small enough $t>0, \gamma_{1}(t), \ldots, \gamma_{k}(t)$ is a $2 \epsilon$-separated net in $X_{t}$. By equicompactness, 
we get $k \leq N_{2}(2 \epsilon)<\infty$. The pseudo metric space $\left(\Lambda^{s u b}, d_{\lim }\right)$ is therefore totally bounded and its completion $\left(X_{\text {lim }}, d_{\text {lim }}\right)$ is a compact metric space (by the theorem of Hausdorff).

We claim that $\left(X_{\lim }, d_{\lim }\right)$ is the Gromov-Hausdorff $\operatorname{limit}_{\lim } \rightarrow 0^{+} X_{t}$. By total boundedness of $\Lambda^{\text {sub }}$, there exists a finite $\epsilon$-dense net $\gamma_{1}, \ldots, \gamma_{k} \in \Lambda^{\text {sub }}$. From the theorem of Kurdyka-Orro we infer the existence of a subanalytic distance $\tilde{d}$ with $\tilde{d} \leq d_{X_{t}} \leq 2 \tilde{d}$ for each $t$. If the subanalytic set $\{(t, x)$ : $\left.x \in X_{t}, \tilde{d}\left(x, \gamma_{i}(t)\right)>2 \epsilon, i=1, \ldots, k\right\}$ contains points with arbitrarily small $t>0$, the curve selection lemma implies that there is a subanalytic curve $\gamma \in \Lambda^{\text {sub }}$ contained in it. But then $d_{\lim }\left(\gamma, \gamma_{i}\right) \geq 2 \epsilon$ for $i=1, \ldots, k$ which is a contradiction. It follows that $\gamma_{1}(t), \ldots, \gamma_{k}(t)$ form, for $t$ sufficiently small, a $4 \epsilon$-dense net in $X_{t}$.

Since the Gromov-Hausdorff distance between a compact metric space and an $\epsilon$-dense net is at most $\epsilon$, and since the Gromov-Hausdorff distance between $\left(\left\{\gamma_{1}, \ldots, \gamma_{k}\right\}, d_{\lim }\right)$ and $\left(\left\{\gamma_{1}(t), \ldots, \gamma_{k}(t)\right\}, d_{X_{t}}\right)$ tends to 0 , the triangle inequality implies that $d_{G-H}\left(X_{\lim }, X_{t}\right) \rightarrow 0$ for $t \rightarrow 0$.

Now we consider more closely the case of the tangent space. Let $X \subset \mathbb{R}^{n}$ be a closed subanalytic subset, $x \in X$. Without loss of generality we assume $x=0$. Define $Y \subset \mathbb{R} \times \mathbb{R}^{n}$ as the set of all points $y=(t, x)$ with $t>0$ and $\frac{1}{t} x \in X$. For $r>0$ denote by $Y^{r}$ the subset of all $y=(t, x) \in Y$ with $\left\|\frac{1}{t} x\right\| \leq r$. The fiber $Y_{t}^{r}$ of the family $Y^{r}$ is just the ball $B_{r t} \subset X$ with the metric rescaled by $\frac{1}{t}$. By the local conical structure of $X$, it is connected for each $r>0$ and all sufficiently small $t$. Therefore the spaces $Y_{t}^{r}$ considered with the inner metric are equicompact.

This and the proof of Theorem 1.1 above show, that the pointed spaces $\left(Y_{t}, d_{Y_{t}},(t, 0)\right)=\left(X, \frac{1}{t} d_{X}, x\right)$ converge in the pointed Gromov-Hausdorff topology to the space $\Lambda$ of all continuous subanalytic curves $\gamma$ with $\gamma(t) \in Y_{t}$, where the metric is defined as in the proof above.

Let $\Lambda_{x}^{s u b} X$ be the set of all continuous subanalytic curves in $X$ starting in $x$ such that $\lim _{t \rightarrow 0} \frac{\|\eta(t)-x\|}{t}<\infty$. Considering the map $\Lambda \rightarrow \Lambda_{x}^{s u b} X$ defined by $\gamma \rightarrow \eta: \eta(t)=t \gamma(t) \in X$, we conclude from the arguments above, that $d\left(\eta_{1}, \eta_{2}\right):=\lim _{t \rightarrow 0} \frac{d_{X}\left(\eta_{1}(t), \eta_{2}(t)\right)}{t}$ defines a metric on $\Lambda_{x}^{s u b} X$ and that the spaces $\left(X, \frac{1}{t} d_{X}, x\right)$ converge to $\Lambda_{x}^{s u b} X$ in the pointed Gromov-Hausdorff topology.

As was already mentioned in the introduction we get more than just an abstract Gromov-Hausdorff convergence.

Corollary 3.1. Let $x \in X$, where $X$ is closed subanalytic. Then the tangent cone of $\left(X, d_{X}\right)$ at the point $x$ exists and is given by completion of the pseudo-

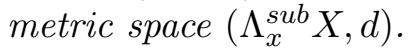

Proof. The dilatations on $\Lambda_{x}^{s u b} X$ are given by linear reparameterizations and induce natural dilatations on $T_{x} X$. 
The "exponential maps" $\exp ^{\left(t_{i}\right)}: \Lambda_{x}^{s u b} X \rightarrow X_{x}^{\left(t_{i}\right)}, \eta \mapsto\left(\eta\left(t_{i}\right)\right)$ extend to isometries on the completion $T_{x} X$. The commutation relations required in Subsection 2.4 are obviously satisfied.

Observe that each $\gamma \in \Lambda_{x}^{s u b} X$ starting at $x$ has a well defined initial direction $v \in T_{x} X$ (i.e. $\gamma$ is differentiable at 0 as a map from the interval $[0, \epsilon]$ to $\left(X, d_{X}\right)$ if $\gamma$ is Lipschitz).

Let now $f:(X, x) \rightarrow(Y, y)$ be a subanalytic Lipschitz map (with respect to the inner metrics) between subanalytic sets. One gets a well defined homogeneous Lipschitz differential $D_{x} f: \Lambda_{x}^{s u b} X \rightarrow \Lambda_{y}^{s u b} Y, \gamma \mapsto f \circ \gamma$ which extends to a Lipschitz differential $D_{x} f: T_{x} X \rightarrow T_{y} Y$.

\section{Regularity of GeOdesics}

Let $X \subset \mathbb{R}^{n}$ be a closed subanalytic set, $x \in X$ a point.

Lemma 4.1. There are $C, \alpha, r>0$ (depending on $X$ and $x$ ) such that for each $z \in X$ with $\|z-x\| \leq r$ there is a Lipschitz curve $\gamma$ in $X$ of length at most $\|z-x\|+C\|z-x\|^{1+2 \alpha}$ connecting $x$ and $z$.

Proof. Stratify $X$ such that Whitney's condition A is satisfied for each pair of strata. Consider on $X \backslash\{x\}$ the stratified vector field $V$ such that $V(y)$ is the projection of $\frac{x-y}{\|x-y\|}$ onto $T_{y} S$, where $S$ is the stratum containing $y$. Of course $\|V(y)\| \leq 1$. Define the subanalytic function $g(t):=\sup \left\{\left\|V(y)-\frac{x-y}{\|x-y\|}\right\|: y \in X, y \neq x,\|y-x\| \leq t\right\}$. If $g(t)$ does not tend to 0 for $t \rightarrow 0$, there is a sequence $\left(y_{i}\right)$ tending to $x$ contained in one single stratum $S$ such that the angle between the tangent space $T_{y_{i}} S$ and the line between $x$ and $y_{i}$ does not tend to 0 , in contradiction to Whitney's condition A. By Łojasiewicz' inequality we get $g(t) \leq C_{1} t^{2 \alpha}$ for some constants $\alpha, C_{1}>0$.

Consider now a maximal integral curve $\gamma$ of $V$ starting at $z$ in the stratum containing $z$. It converges to a unique point $z_{1}$ in a stratum of smaller dimension. Then continue on the maximal integral curve of $V$ starting in $z_{1}$ and so on. After finitely many steps we get a Lipschitz curve $\gamma$ connecting $z$ and $x$. Let $s$ be the smallest real such that $\gamma(s)=0$ and set $\bar{\gamma}(t):=$ $\gamma(s-t), t \in[0, s]$. From $\|V(y)\| \leq 1$ we get $L(\bar{\gamma})=L(\gamma) \leq s$ and $f(t):=$ $\|\bar{\gamma}(t)-x\| \leq t$ for $t \in[0, s]$.

Then

$$
\begin{aligned}
\frac{d}{d t} f^{2} & =2\left\langle\bar{\gamma}^{\prime}(t), \bar{\gamma}(t)-x\right\rangle \\
& =2\left\langle-V(\bar{\gamma}(t))+\frac{x-\bar{\gamma}(t)}{\|x-\bar{\gamma}(t)\|}, \bar{\gamma}(t)-x\right\rangle-2\left\langle\frac{x-\bar{\gamma}(t)}{\|x-\bar{\gamma}(t)\|}, \bar{\gamma}(t)-x\right\rangle \\
& \geq 2 f(t)-2 C_{1} t^{2 \alpha} f(t)
\end{aligned}
$$

We conclude that (for $t>0) f^{\prime}(t) \geq 1-C_{1} t^{2 \alpha}$ and therefore $\|z-x\|=$ $f(s)=\int_{0}^{s} f^{\prime}(t) d t \geq s-C_{2} s^{1+2 \alpha}$ with $C_{2}:=\frac{C_{1}}{1+2 \alpha}$. 
For $s$ sufficiently small, $C_{2} s^{2 \alpha} \leq \frac{1}{2}$. Replacing this yields $s \leq 2\|z-x\|$ and finally $L(\gamma) \leq s \leq\|z-x\|+C\|z-x\|^{1+2 \alpha}$ with $C:=2^{1+2 \alpha} C_{2}$.

Remark 4.1. This lemma can be reformulated in terms of the identity map $\iota:\left(X, d_{X}\right) \rightarrow\left(X, d_{e}\right)$ as follows. For each $z \in X$ holds $d_{e}(\iota(z), \iota(x)) \geq$ $d_{X}(x, z)-C d_{X}(x, z)^{1+2 \alpha}$, for some $C, \alpha$ depending on $x$. Moreover we see that by the pointed Gromov-Hausdorff convergence $\left(X, \frac{1}{t} d_{X}, x\right) \rightarrow T_{x} X$ the intersections $\left(S_{t}(x), \frac{1}{t} d_{X}\right)$ of the Euclidean spheres with $X$ converge to the unit sphere in $S$ in $T_{x} X$.

Now we derive:

PROPOSITION 4.2. Let $\gamma:[0, t] \rightarrow X$ be a geodesic starting at $x$. Then $\gamma$, considered as a curve in $\mathbb{R}^{n}$, has a unique direction $\gamma^{+}$at 0 . Moreover, there are $r, C, \alpha>0$ depending only on $X$ and $x$ such that for all $0<t \leq r$ $\left\|\gamma^{+}-\frac{\gamma(t)-x}{\|\gamma(t)-x\|}\right\| \leq C t^{\alpha}$.

Proof. We choose $r, C, \alpha$ as in Lemma 4.1 and $0<t \leq r$. Let first $s$ be a number with $\frac{t}{2} \leq s \leq t$. Put $z=\gamma(t)$ and $y=\gamma(s)$. In the triangle $x y z$ we know $\|x-y\| \leq s,\|y-z\| \leq t-s$ and $\|x-z\| \geq t-C t^{1+2 \alpha}$, with $C=C(X, x)$.

Using the cosine law for the (Euclidean) triangle $x y z$ we get that the angle at $x$ between $x z$ and $x y$ is at most $\bar{C} t^{\alpha}$, for some $\bar{C}=\bar{C}(C)$.

Thus the directions $v_{t}:=\frac{\gamma(t)-x}{\|\gamma(t)-x\|}$ satisfy $\left\|v_{t}-v_{s}\right\| \leq C t^{\alpha}$ for each $t>s \geq$ $\frac{t}{2}$. From this we immediately conclude that $v_{t}$ converge for $t \rightarrow 0$ to some $v$. Moreover we get $\left\|v_{t}-v\right\| \leq \sum_{i=0}^{\infty}\left\|v_{2^{-i} t}-v_{2^{-i-1} t}\right\| \leq C t^{\alpha} \sum_{i=0}^{\infty}\left(2^{\alpha}\right)^{-i}=\tilde{C} t^{\alpha}$, with some $\tilde{C}$ depending on $C$ and $\alpha$.

\section{Comparison Between the Metric And the Subanalytic TANGENT CONE}

The natural embedding $\iota_{X}:\left(X, d_{X}\right) \rightarrow \mathbb{R}^{n}$ is subanalytic and 1-Lipschitz. Hence $\iota_{X}$ is differentiable at $x$ with differential $D_{x} \iota_{X}: T_{x} X \rightarrow T_{x} \mathbb{R}^{n}=\mathbb{R}^{n}$ (see Section 3). The image $D_{x} \iota_{X}\left(T_{x} X\right)$ coincides with $T_{x}^{s u b} X$. Due to Remark 4.1, $D_{x} \iota_{X}$ preserves distances to the origin. If $f:(X, x) \rightarrow(Y, y)$ is a subanalytic Lipschitz map with respect to the induced metrics, then $f$ is also Lipschitz with respect to the length metrics and the differentials commute, i.e. $D_{x}^{s u b} f \circ D_{x} \iota X=D_{y} \iota_{Y} \circ D_{x} f$.

Fix $\epsilon>0$ and let again $X=\cup_{j=1}^{m} X_{j}$ be a decomposition in subanalytic sets such that inner and induced metric agree up to a factor $1+\epsilon$ on each of it. The injection $\tau_{j}: X_{j} \rightarrow X$ is subanalytic, hence it induces a 1Lipschitz differential $D_{x} \tau_{j}: T_{x} X_{j} \rightarrow T_{x} X$. Denote by $\tilde{T}_{x} X_{j}$ the image $D_{x} \tau_{j}\left(T_{x} X_{j}\right) \subset T_{x} X$. Remark that $T_{x} X=\cup_{j=1}^{m} \tilde{T}_{x} X_{j}$.

Since the restriction of $d_{X}$ to $X_{j}$ is $(1+\epsilon)$-bilipschitz equivalent to the induced metric on $X_{j}$, the map $D_{x} \iota x: \tilde{T}_{x} X_{j} \rightarrow D_{x} \iota_{X}\left(\tilde{T}_{x} X_{j}\right)$ is $(1+\epsilon)$ bilipschitz. In particular this restriction is injective. This shows that all 
fibers of the map $D_{x} \iota_{X}: T_{x} X \rightarrow T_{x}^{s u b} X$ have at most $m$ elements. Moreover $\tilde{T}_{x} X$ has a finite decomposition such that each set of this decomposition is mapped $(1+\epsilon)$-bilipschitz under $D_{x} \iota X$ onto its image in $T_{x}^{s u b} X$. Since this holds for each $\epsilon>0$ we see that $D_{x} \iota \iota_{X}$ preserves lengths of curves. These observations complete the proof of Theorem 1.4.

Now we are going to prove that the tangent cone $T_{x} X$ is a Euclidean cone. This result is a direct consequence of Theorem 1.4, the fact that $T_{x}^{s u b} X$ is a Euclidean cone as a subcone of $\mathbb{R}^{n}$ and the following:

Lemma 5.1. Let $T$ be a metric cone that is in addition a geodesic metric space. Let $\iota: T \rightarrow C V$ be a homogeneous 1-Lipschitz arclengths preserving map onto an Euclidean cone $C V$, that preserves the distance to the origin. Then $T$ is an Euclidean cone.

Proof. Let $S$ be the unit sphere in $T$. It is mapped by $\iota$ to $V$. For $x \in S$ the image $\iota\left(\delta_{t}(x)\right)=\delta_{t}(\iota(x))$ is a radial ray in $C V$. Since $\iota$ preserves the lengths of curves we see that $\delta_{t}(x)$ and $\delta(s)(x)$ have distance at most $|s-t|$. Therefore $T$ is a radial cone.

The restriction $\iota: S \rightarrow V$ is again 1-Lipschitz and preserves lengths of curves. Let $\tilde{S}$ be the set $S$ considered with the inner metric. Consider the Euclidean cone $C \tilde{S}$ and the natural bijection $F: C \tilde{S} \rightarrow T$. Observe that the composition $\iota \circ F: C \tilde{S} \rightarrow C V$ preserves the lengths of curves. Since so does the map $\iota: T \rightarrow C V$ and since $C \tilde{S}$ and $T$ are geodesic metric spaces, it is enough to see that the bijection $F$ preserves the class of Lipschitz curves. But so does the natural 1-Lipschitz map $I d: C \tilde{S} \rightarrow C S$ and by Subsection 2.2 the natural map $F: C S \rightarrow T$ is bilipschitz. This finishes the proof.

\section{Connectivity}

Proof of Theorem 1.3, a). Let $X$ be a closed subanalytic space, $x \in X$. By local conical structure of $X$, the (Euclidean) ball $B_{r}(x)$ around $x$ is homeomorph to the cone over the (Euclidean) sphere $S_{r}(x)$ for $r>0$ small enough. Suppose that $S_{r}(x)$ is not connected and let $S_{1}, S_{2}, \ldots, S_{k}$ be its connected components. They correspond to connected components $B_{1}, \ldots, B_{k}$ of $B_{r}(x) \backslash\{x\}$. Since each $d_{X}$-geodesic between points from different components $B_{i}$ and $B_{j}$ must run through $x$, we see that for the closed subcones $T_{x} B_{i}$ of $T_{x} X$ holds $T_{x} B_{i} \cap T_{x} B_{j}=\{0\}$. Therefore $T_{x} B_{i} \backslash\{0\}$ is open and closed in $T_{x} X \backslash\{0\}$ and the unit sphere in $T_{x} X$ is not connected.

Assume on the other hand that $S_{r}$ is connected for small $r$. Observe that by the convergence $\left(X, \frac{1}{t} d_{X}, x\right) \rightarrow T_{x} X$ the spheres $\left(S_{r}, \frac{1}{r} d_{X}\right)$ converge to the unit sphere in $T_{x} X$. But $\frac{1}{r} S_{r}$ is a subanalytic family of bounded connected subanalytic subsets for $r>0$. By the equicompactness of the family (see Section 3) the fibers $S_{r}$ of the family have uniformly bounded diameters with respect to the inner metrics $d_{S_{r}}$. Hence the unit sphere in $T_{x} X$ is connected. 
For a vector $v \in \mathbb{R}^{n}$ and $\rho>0$ denote by $K(v, \rho)$ the set of all vectors $w \in \mathbb{R}^{n}$ with $\langle v, w\rangle \geq(1-\rho)\|v\|\|w\|$. In the next section we will use:

Lemma 6.1. Let $X$ be a subanalytic space, $0 \in X$. Let $v \in T_{0}^{s u b} X$ be a direction. For the canonical map $D_{0} \iota_{X}: T_{0} X \rightarrow T_{0}^{\text {sub }} X$ let $I$ be the finite set $D_{0} \iota_{X}^{-1}(v)$. Then for some $\rho>0$ the intersection $Y$ of $X$ with the cone $K(v, \rho)$ has the property, that different points of I lie in different components of $T_{0} Y \backslash\{0\}$

Proof. If $v=0$ then $D_{0} \iota_{X}^{-1}(v)=\{0\}$ and the claim is trivial. If $v \neq 0$ we may assume that $\|v\|=1$. Let $5 s$ be the minimal distance of two points in $I$. Let $U_{1}, U_{2}, \ldots$ be a sequence of neighborhoods of $v$ in $S^{s u b}$ with diameters tending to 0 . If for each $i=1,2, \ldots$ there exists a point $w_{i} \in D_{0} \iota_{X}^{-1}\left(U_{i}\right)$ with $d\left(w_{i}, I\right) \geq s$, we can (by compactness of the unit sphere $S \subset T_{x} X$ ) extract a converging subsequence of $\left(w_{i}\right)$. If $w$ denotes its limit, then $d(w, I) \geq s$ and $D_{0} \iota_{X}(w)=v$, contradiction. Therefore, for some $i$, the intersections of $D_{0} \iota_{X}^{-1}\left(U_{i}\right)$ with balls of radius $2 s$ around points of $I$ are open and closed in $D_{0} \iota_{X}^{-1}\left(U_{i}\right)$. Taking $\rho$ so small that $K(v, \rho) \cap S^{s u b}$ is contained in this neighborhood $U_{i}$, we obtain the result.

\section{Finer properties of the tangent CONE}

Now we are going to compare the tangent cone with the Alexandrov cone at the given point. We start with:

Lemma 7.1. Let $X$ be a closed connected subanalytic space and suppose $0 \in X$. Let $\gamma$ be a Lipschitz curve in $X$ starting at 0 , that has a Euclidean differential $v \in T_{0}^{s u b} X$ at 0 . Then $\gamma$ is also differentiable at 0 as a curve into $\left(X, d_{X}\right)$

Proof. Consider the cone $K(v, \rho)$ as in Lemma 6.1 and set $Y=X \cap K(v, \rho)$. Then a small starting part of $\gamma$ is contained in $Y$ and it is sufficient to prove that $\gamma$ is differentiable at 0 as map into $\left(Y, d_{Y}\right)$. We may assume that $\|v\| \neq 0$. Then a beginning part of $\gamma$ is contained in a connected component $C$ of $\left(B_{r}(0) \cap Y\right) \backslash\{0\}$. By Theorem 1.3 a) and Lemma 6.1 the restriction $\iota: T_{0} C \rightarrow T_{0}^{s u b} C$ has the property that $i^{-1}(v)$ has only one point $w$. But for each zero sequence $t_{i}$ the point $\left(\gamma\left(t_{i}\right)\right) \in C_{0}^{\left(t_{i}\right)}=T_{0} C$ is mapped by $\iota$ onto $v$. Hence $\left(\gamma\left(t_{i}\right)\right)=w$ and we are done.

Together with Proposition 4.2 this shows that geodesics are differentiable as maps into $\left(X, d_{X}\right)$. In particular for geodesics $\gamma_{1}$ and $\gamma_{2}$ starting at $x$ and for each $s \geq 0$ the generalized angle $\lim _{t \rightarrow 0} \frac{d\left(\gamma_{1}(s t), \gamma_{2}(t)\right)}{t}$ is well defined. Hence the exponential maps $\exp _{x}^{\left(t_{i}\right)}: C_{x} X \rightarrow T_{x} X$ are isometric embeddings. Since $T_{x} X$ is a Euclidean cone, the same is true for $C_{x} X$ and the usual angle between geodesics is well defined too.

The next lemma finishes the proof of Theorem 1.2: 
Lemma 7.2. In the above notations the exponential maps $\exp _{x}^{\left(t_{i}\right)}: C_{x} X \rightarrow$ $X_{x}^{\left(t_{i}\right)}=T_{x} X$ are surjective.

Proof. It is enough to prove that for a curve $\eta \in \Lambda_{x}^{s u b}$, with starting direction $v$ in the unit sphere $S$ of $T_{x} X$, a geodesic $\gamma_{n}$ between $x$ and $\eta\left(\frac{1}{n}\right)$ with starting direction $v_{n} \in S$ holds $v_{n} \rightarrow v$. Consider again the intersection $Y$ of $X$ with a small cone $K(\iota(v), \rho)$ as in the last lemma. Let $C$ be the component of $\left(B_{r}(x) \cap Y\right) \backslash\{x\}$ containing $\eta$. Due to Lemma 4.1 the geodesics $\gamma_{n}$ are contained in $Y$ for big $n$. Moreover $\iota\left(v_{n}\right)$ converge to $\iota(v)$. Hence for each limit point $w$ of $v_{n}$ holds $\iota(w)=\iota(v)$. Since $v$ is the only preimage point of $\iota(v)$ in $T_{0} C$, we obtain $v=w$ and finish the proof.

Remark 7.1. The (natural) equality between the tangent cone $T_{x} X$ and the Alexandrov cone $C_{x} X$ implies directly (compare [15]), that each isometry between subanalytic spaces is differentiable at each point. This reflects the fact, that the tangent cones, although they are defined in subanalytic terms, are in fact purely metric invariants of $X$, not only as isometry classes (which is trivial) but as metric spaces.

\section{Measure And Dimension}

Let $X$ be a compact subanalytic set. Then due to the decomposition of $X$ in pieces where $d_{X}$ and $d_{e}$ coincide up to some factor near 1 , we see that the identity $\iota:\left(X, d_{x}\right) \rightarrow\left(X, d_{e}\right)$ preserves the Hausdorff measures $\mathcal{H}^{l}$, for each $l \in \mathbb{R}^{+}$.

On the other hand the Hausdorff dimension $k$ of $X$ coincides with its topological dimension and is given by the dimension of the maximal stratum in a stratification of $X$. Moreover the $k$-dimensional Hausdorff measure is finite on bounded subsets and is positive on each $k$-dimensional subanalytic subset.

The same statements hold true for the subanalytic tangent cone $T_{x}^{s u b}$. Since $T_{x} X$ has a finite decomposition such that $D_{x} \iota: T_{x} X \rightarrow T_{x}^{s u b} X$ is almost 1-bilischitz on pieces of the decomposition, we see that the Hausdorff dimension of $T_{x} X$ coincides with the Hausdorff dimension of $T_{x}^{s u b} X$. Moreover if the restriction of $D_{x} \iota$ onto a subset $U$ of $T_{x} X$ is injective, then $D_{x} \iota: U \rightarrow D_{x} \iota(U)$ preserves the Hausdorff measure. Finally restricting $\iota$ to the preimage of a maximal stratum of a stratification of $T_{x}^{s u b} X$, we see that $T_{x} X$ contains an open subset homeomorph to a $\operatorname{dim}\left(T_{x}^{s u b} X\right)$ - dimensional ball. Hence the topological dimension of $T_{x} X$ coincides with its Hausdorff dimension.

Proof of Theorem 1.3, b). Choose $\epsilon>0$ and a decomposition $X=\cup_{i=1}^{m} X_{i} \cup$ $Y$ such that $X_{1}, \ldots, X_{m}$ are $\epsilon$-analytic pieces ([14]) and $\operatorname{dim} Y<k$. Then $\operatorname{dim}\left(T_{x} Y\right)=\operatorname{dim}\left(T_{x}^{s u b} Y\right) \leq \operatorname{dim} Y<k$. Since $T_{x} X_{i} \cap T_{x} X_{j} \subset T_{x} X$ is contained in $T_{x} Y$ it is therefore enough to prove the proposition in the case where $X$ is the closure of a single subset $X_{i}$. In this case $T_{x} X=T_{x}^{s u b} X$ and the result was shown in ([14], Proposition 3.6. and Theorem 3.8). 
Remark 8.1. Suppose that $v \in T_{x}^{s u b} X$ is moreover contained in the pure tangent cone ([14]). Then the multiplicity $n(x)$ defined as in [14] equals the cardinality of the fiber of the map $D_{x} \iota: T_{x} X \rightarrow T_{x}^{s u b}$ above $v$. This follows by a similar argument as above.

\section{Semialgebraic metric Spaces}

In a naive sense, the Gromov-Hausdorff limit of a semialgebraic family will not be semialgebraic. Consider for instance a family of ellipsoids getting thinner and thinner. The limit space is a double disc, which is not isometric to the semialgebraic limit consisting of a single disc. But we will show that such a limit space can be obtained as inner metric space associated to a semialgebraic set over some real closed field $R$.

For the notions of semialgebraic sets, real spectrum and fiber of a semialgebraic family over a point in the real spectrum we refer to [5].

Let $R$ be a real closed field. Let $A$ be the convex hull of $\mathbf{Z} \subset R$. Then $A$ is a valuation ring of $R$. We denote by $m_{A}$ its maximal ideal and by $\pi: A \rightarrow A / m_{A}$ the canonical projection. The field $A / m_{A}$ is archimedean and can therefore be uniquely identified with a subfield of $\mathbb{R}$. We define the real place $\lambda_{R}: R \rightarrow \mathbb{R} \cup\{\infty\}$ by setting $\lambda_{R}(x)=\infty$ if $x \notin A$ and $\lambda_{R}(x):=\pi(x)$ if $x \in A$.

An alternative way to define $\lambda_{R}$ is given by $\lambda_{R}(x)=\inf \{r \in \mathbf{Q}: r>x\}$ (with the convention $\inf \emptyset=\inf \mathbf{Q}=\infty$ ).

Let $S \subset R^{n}$ be a closed connected semialgebraic set. A path in $S$ is a continuous map $\gamma:[0,1] \rightarrow S$, where $[0,1] \subset R$ is the closed unit interval in $R$. The length of $\gamma$ is defined by

$$
l(\gamma):=\sup \left\{\lambda_{R}\left(\sum_{i=0}^{k-1}\left\|\gamma\left(t_{i+1}\right)-\gamma\left(t_{i}\right)\right\|\right): 0=t_{0}<t_{1}<\ldots<t_{k}=1\right\}
$$

The distance between two points $x, y \in S$ is defined by

$$
d_{S}(x, y):=\inf \{l(\gamma): \gamma \text { is a path between } x \text { and } y\} \in \mathbb{R} \cup\{\infty\}
$$

Note that this is a real number and not a number in $R$.

Definition 9.1. A metric space $(X, d)$ is called semialgebraic if there exists a real closed field $R$, an integer $n$, a closed connected semialgebraic set $S \subset$ $R^{n}$ such that $(X, d)$ equals the completion of $\left(S, d_{S}\right)$.

Proof of Theorem 1.5. We first construct a semialgebraic set $S$ and show afterwards that it is the Gromov-Hausdorff limit of the family. Let $R^{\prime}:=$ $R(t)_{a l g}^{\wedge}$ denote the (real closed) field of algebraic Puiseux-series in the parameter $t$. Equivalently, $R^{\prime}$ is the real closed field associated to the point $0_{+}$ in the real spectrum of $R[t]$.

An element $\gamma \in R^{\prime}$ can be identified with the germ at $0_{+}$of a continuous semialgebraic curve $\gamma:(0, \epsilon) \rightarrow R(\epsilon \in R)$. 
Let $S:=X_{0^{+}} \subset\left(R^{\prime}\right)^{n}$ be the fiber of $X$ above $0^{+} . S$ consists of those semialgebraic curve germs with $\gamma(t) \in X_{t}$ for all sufficiently small $t>0$. We will show that $\left(\bar{S}, \bar{d}_{S}\right)$ is the Gromov-Hausdorff limit of the family $X$.

Lemma 9.1. Let $X \subset R \times R^{n}$ be a semialgebraic family which is closed, rationally bounded (i.e. there exists a natural number a with $X \subset B_{a}(0)$ ) and fiberwise semialgebraically connected. Then, for any rational number $C>1$, there exists a decomposition $X=\cup_{i=1}^{m} X^{i}$ such that each $X_{t}^{i}$ is connected and such that for $x, y \in X_{t}^{i}$

$$
\lambda_{R}(\|x-y\|) \leq d_{X_{t}^{i}}(x, y) \leq C \lambda_{R}(\|x-y\|)
$$

and such that for $\gamma_{1}, \gamma_{2} \in X_{0^{+}}^{i}$

$$
\lambda_{R^{\prime}}\left(\left\|\gamma_{1}-\gamma_{2}\right\|\right) \leq d_{S^{i}}\left(\gamma_{1}, \gamma_{2}\right) \leq C \lambda_{R^{\prime}}\left(\left\|\gamma_{1}-\gamma_{2}\right\|\right) .
$$

The proof of the lemma is by extending the proof contained in [13] (which is based on [12]) to arbitrary real closed field. In most parts of the proof, one can just replace $R$ by $\mathbb{R}$. This is not the case for the compactness of the Grassmannians used in [12], but this can be easily replaced by model completeness.

We continue the proof of the theorem. Let $\epsilon>0$ be a rational number. Apply the above Lemma (with $C:=2$ ) to $X$. Let $x_{1}, \ldots, x_{k} \in X_{t}^{i}$ be an $\epsilon$-separated net (with respect to $\left.d_{X_{t}}\right)$. Then, if $j_{1} \neq j_{2}, \lambda_{R}\left(\left\|x_{j_{1}}-x_{j_{2}}\right\|\right) \geq \frac{\epsilon}{2}$ which implies that $\left\|x_{j_{1}}-x_{j_{2}}\right\| \geq \frac{\epsilon}{4}$.

The size of an $\frac{\epsilon}{4}$-separated net in $B(0, a)$ is bounded by a function of $\epsilon$ and $a$. This is trivial if $R=\mathbb{R}$ (by considering the volume) and follows by model completeness for all real closed $R$.

Therefore, the size of an $\epsilon$-separated net in $X_{t}$ is bounded by a number which only depends on $\epsilon, a, m$, but not on $t$. It follows that the family of pseudo-metric spaces $\left(X_{t}, d_{X_{t}}\right)$ is equicompact. In particular, each $\left(X_{t}, d_{X_{t}}\right)$ is totally bounded, which implies (by the theorem of Hausdorff) that the completion $\left(\bar{X}_{t}, \bar{d}_{X_{t}}\right)$ is compact.

By the same reasoning, the space $\left(S, d_{S}\right)$ is totally bounded and its completion $\left(\bar{S}, \bar{d}_{S}\right)$ compact.

Choose a rational $C>1$ and a decomposition $X=\cup_{i=1}^{m} X^{i}$ as in the lemma. Similarly as in [13], we define a semialgebraic function $\tilde{d}: X \times X \rightarrow$ $R$ by $\tilde{d}(x, y):=0$ if $x$ and $y$ lie in different fibers and

$\tilde{d}(x, y):=\inf \left\{\sum_{i=0}^{m^{\prime}-1}\left\|x_{i}-x_{i+1}\right\|: x=x_{0}, x_{1}, \ldots, x_{m^{\prime}}=y\right.$ is a chain, $\left.m^{\prime} \leq m\right\}$

Here the word "chain" means that two consecutive of the $x_{i}$ lie in the closure of one of the $X_{t}^{i}$ (where $t$ is fixed). It is clear from the definition that $\tilde{d}$ is bounded by the natural number $2 m a$.

By the lemma, we get for $x, y \in X_{t}$

$$
\lambda_{R}(\tilde{d}(x, y)) \leq d_{X_{t}}(x, y) \leq C \lambda_{R}(\tilde{d}(x, y))
$$


and for $\gamma_{1}, \gamma_{2} \in S=X_{0^{+}}$

$$
\lambda_{R^{\prime}}\left(\tilde{d}\left(\gamma_{1}, \gamma_{2}\right)\right) \leq d_{S}\left(\gamma_{1}, \gamma_{2}\right) \leq C \lambda_{R^{\prime}}\left(\tilde{d}\left(\gamma_{1}, \gamma_{2}\right)\right) .
$$

Let $\gamma_{1}, \gamma_{2}$ be two points in $X_{0^{+}}$. Using the fact that the limit $\lim _{t \rightarrow 0^{+}} \tilde{d}\left(\gamma_{1}(t), \gamma_{2}(t)\right) \in R$ exists (since $\tilde{d}$ is bounded by $2 m a$ and semialgebraic and $\gamma_{1}, \gamma_{2}$ are semialgebraic) and using the alternative description of $\lambda_{R}, \lambda_{R^{\prime}}$, we obtain

$$
\lim _{t \rightarrow 0^{+}} \lambda_{R}\left(\tilde{d}\left(\gamma_{1}(t), \gamma_{2}(t)\right)=\lambda_{R^{\prime}}\left(\tilde{d}\left(\gamma_{1}, \gamma_{2}\right)\right) .\right.
$$

From this we conclude

$$
\frac{1}{C} \limsup _{t \rightarrow 0^{+}} d_{X_{t}}\left(\gamma_{1}(t), \gamma_{2}(t)\right) \leq d_{S}\left(\gamma_{1}, \gamma_{2}\right) \leq C \liminf _{t \rightarrow 0^{+}} d_{X_{t}}\left(\gamma_{1}(t), \gamma_{2}(t)\right) .
$$

Since $C$ was an arbitrary rational number with $C>1$, it follows that

$$
d_{S}\left(\gamma_{1}, \gamma_{2}\right)=\lim _{t \rightarrow 0^{+}} d_{X_{t}}\left(\gamma_{1}(t), \gamma_{2}(t)\right) .
$$

Now we continue the proof as in Section 3 (replacing "subanalytic" by "semialgebraic") to see that $\left(\bar{S}, \bar{d}_{S}\right)$ equals the Gromov-Hausdorff limit $\lim _{t \rightarrow 0^{+}}\left(\bar{X}_{t}, \bar{d}_{X_{t}}\right)$.

Remark 9.1. The above proof, applied to a constant family, shows that the metric space associated to a rationally bounded, connected, closed semialgebraic set is compact. Since the Gromov-Hausdorff distance between two compact metric spaces vanishes if and only if they are isometric, the metric space associated to an extension of a semialgebraic $S$ to another real closed field ([5]) gives rise to the same metric space.

Remark 9.2. The Hausdorff limit of the family $X$ at $0^{+}$is given by $\lambda_{R}(S)$, endowed with the Euclidean metric ([7]). This shows that tangent cone and semialgebraic tangent cone at a point $x$ of a closed semialgebraic set are given by the same semialgebraic set, but the former gets the length metric and the latter gets the Euclidean metric.

\section{REFERENCES}

[1] A. Bernig, Scalar Curvature of definable Alexandrov spaces. Advances in Geometry 2 (2002), 29-55.

[2] A. Bernig, Scalar curvature of definable CAT-spaces. Advances in Geometry 3 (2003), 23-43.

[3] E. Bierstone, P. D. Milman, Semianalytic and subanalytic sets. Inst. Hautes Études Sci. Publ. Math. 67 (1988), 5-42.

[4] L. Birbrair, T. Mostowski, Normal embeddings of semialgebraic sets. Michigan Math. J. 47 (2000), 125-132.

[5] J. Bochnak, M. Coste, M.-F. Roy, Géométrie algébrique réelle. Springer Verlag Berlin, 1987.

[6] M. Bridson, A. Haefliger, Metric Spaces of Non-Positive Curvature. Springer-Verlag Berlin-Heidelberg 1999. 
[7] L. Bröcker, Families of semialgebraic sets and limits. In: Real Algebraic Geometry, LNM 1524, Springer-Verlag Berlin 1992, 145-162.

[8] L. Bröcker, M. Kuppe, W. Scheufler, Inner metric properties of 2-dimensional semialgebraic sets. Rev. Mat. Univ. Complut. Madrid 10 (1997), 51-78.

[9] D. Burago, Yu. Burago, S. Ivanov, A course in metric geometry. American Mathematical Society, Providence, RI 2001.

[10] M. Coste, M. Reguiat, Trivialités en famille. In: Real Algebraic Geometry, LNM 1524, Springer-Verlag Berlin 1992, 193-204.

[11] R. Hardt, Some analytic bounds for subanalytic sets. In: Differential geometric control theory, Progress in Math., vol 27, 1983, 259-267.

[12] K. Kurdyka, On a subanalytic stratification satisfying a Whitney property with exponent 1. In: Real Algebraic Geometry, LNM 1524, Springer-Verlag Berlin 1992, 316-322.

[13] K. Kurdyka, P. Orro, Distance géodésique sur un sous-analytique. Rev. Mat. Univ. Complut. Madrid 10 (1997), 173-182.

[14] K. Kurdyka, G. Raby, Densité des ensembles sous-analytiques. Ann. Inst. Fourier 39 (1989), 753-771.

[15] A. Lytchak, Differentiation in metric space. Preprint 2003.

[16] P. Orro, Quelques propriétés de la distance géodésique. In: Real analytic and algebraic singularities, Pitman Res. Notes Math. Ser. 381, Longman, Harlow, 1998, 107-113.

[17] A. Parusinsski, Lipschitz stratification of subanalytic sets. Ann. scient. Ec. Norm. Sup. 27 (1994), 661-696.

[18] M. Pflaum, Analytic and Geometric Study of Stratified Spaces. Springer-Verlag Berlin-Heidelberg 2001.

[19] L. Van den Dries, C. Miller, Geometric Categories and o-minimal structures. Duke Math. Journal 84 (1996), 497-540.

Mathematisches Institut, Universität Freiburg, Eckerstr. 1, 79104 Freiburg, GERMANY

E-mail address: andreas.bernig@math.uni-freiburg.de

Mathematisches Institut, Universität Bonn, Beringstr. 1, 53115 Bonn, GerMANY,

E-mail address: lytchak@math.uni-bonn.de 